\title{
Design and performance of a Tesla transformer type relativistic electron beam generator
}

\author{
K K JAIN, D CHENNAREDDY, P I JOHN and Y C SAXENA \\ Plasma Physics Programme, Physical Research Laboratory, Navrangpura, \\ Ahmedabad 380009, India
}

MS received 25 October 1984; revised 29 July 1985

\begin{abstract}
A relativistic electron beam generator driven by an air core Tesla transformer is described. The Tesla transformer circuit analysis is outlined and computational results are presented for the case when the coaxial water line has finite resistance. The transformer has a coupling coefficient of 0.56 and a step-up ratio of 25 . The Tesla transformer can provide $800 \mathrm{kV}$ at the peak of the second half cycle of the secondary output voltage and has been tested up to $600 \mathrm{kV}$. A $100-200 \mathrm{keV}, 15-20 \mathrm{kA}$ electron beam having $150 \mathrm{~ns}$ pulse width has been obtained. The beam generator described is being used for the beam injection into a toroidal device BETA.
\end{abstract}

Keywords. Relativistic electron beam; Tesla transformer; coaxial water line; drift injection technique.

\section{Introduction}

In the last decade, the technology of the intense relativistic electron beam (REB) has received much attention in the field of plasma physics. Intense electron beams have been used for heating of plasma to high temperatures (Korn et al 1973; Roberson 1978; Jain \& John 1984a), for plasma confinement by creating a close field-reversed configuration (Kapetanakos et al 1975; Sethian et al 1978; Jain \& John 1984b), for collective acceleration of ions (Olsen 1975; Roberson et al 1976; Sprangle et al 1976) and for pellet fusion (Yonas et al 1974, 1983, p. 353).

Electron beam generators used for all the above applications consist of a pulsed high voltage source, a pulse forming line and a vacuum field emission diode. The high voltage pulse can be produced using either a Marx generator or a Tesla transformer. In the Marx generator, a high voltage pulse is obtained by charging a number of capacitors in parallel and discharging them in series. Therefore, the Marx generator requires many stacks of unit elements each consisting of a capacitor, a spark gap switch and a charging resistance. Use of a Tesla transormer type of high voltage source instead of a Marx generator offers the following advantages:

(i) higher voltage gain with simpler circuit design and construction;

(ii) only one spark gap switch is needed and its life time is usually longer than that of the Marx circuit due to lower instantaneous current; 
(iii) overvoltages are lower than in the Marx circuit and hence the breakdown problems are easier to overcome;

(iv) possibility of operation at high repetition rates;

(v) overall cost is low.

In this paper, we describe design details and performance of a relativistic electron beam generator, driven with an air core Tesla transformer, to be used for injecting an electron beam into a toroidal machine BETA (Basic Experiments in Toroidal Assembly) and to study the formation of an electron ring and a current drive. The device is schematically represented in figure 1 . The next section is devoted to the Tesla transformer circuit analysis, design and construction details. In $\$ 3$, a coaxial water line, used as a pulse forming line, is described. Design details of the secondary gas switch and the field emission diode are reported in $\$ 4$. In $\$ 5$; the characteristics of operation of the beam generator are given.

\section{Tesla transformer}

\subsection{Circuit analysis}

The equivalent circuit of a Tesla transformer consist of two energy storage capacitors $C_{1}$ and $C_{2}$, coupled by a transformer with primary inductance $L_{1}$ and secondary inductance $L_{2}$, having a coupling co-efficient $K$, as shown in figure 2 . The other element of the circuit is the secondary loss resistance $R$.

The primary energy storage capacitor $C_{1}$ is charged to a voltage $V_{0}$ and then discharged into the circuit through a switch $S$. The equations for primary and secondary circuit can be written as:

$$
\begin{aligned}
L_{1}\left(\mathrm{~d} i_{1} / \mathrm{d} t\right)+\left(1 / c_{1}\right) \int i_{1} \mathrm{~d} t-M\left(\mathrm{~d} i_{2} / \mathrm{d} t\right) & =V_{0} \\
L_{2}\left(\mathrm{~d} i_{2} / \mathrm{d} t\right)+\left(1 / c_{2}\right) \int i_{3} \mathrm{~d} t-M\left(\mathrm{~d} i_{1} / \mathrm{d} t\right) & =0 \\
\left(1 / c_{2}\right) \int i_{3} \mathrm{~d} t & =R i_{4} \\
i_{2} & =i_{3}+i_{4} .
\end{aligned}
$$

Here $M$, the mutual inductance, is equal to $K\left(L_{1} L_{2}\right)^{1 / 2}$.

Case $A$ when $R$ is infinite: In this case, (4) becomes

$$
i_{2}=i_{3} \text {. }
$$

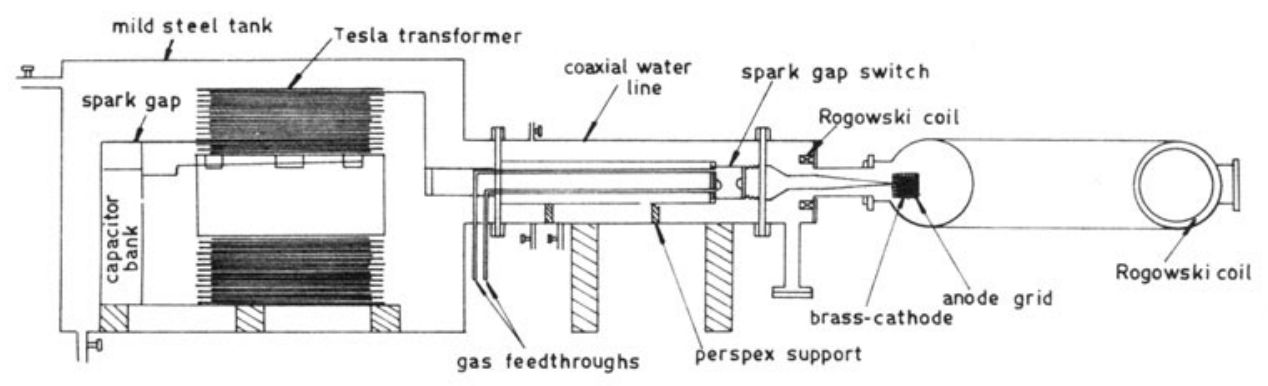

Figure 1. A schematic layout of the relativistic electron beam generator. 


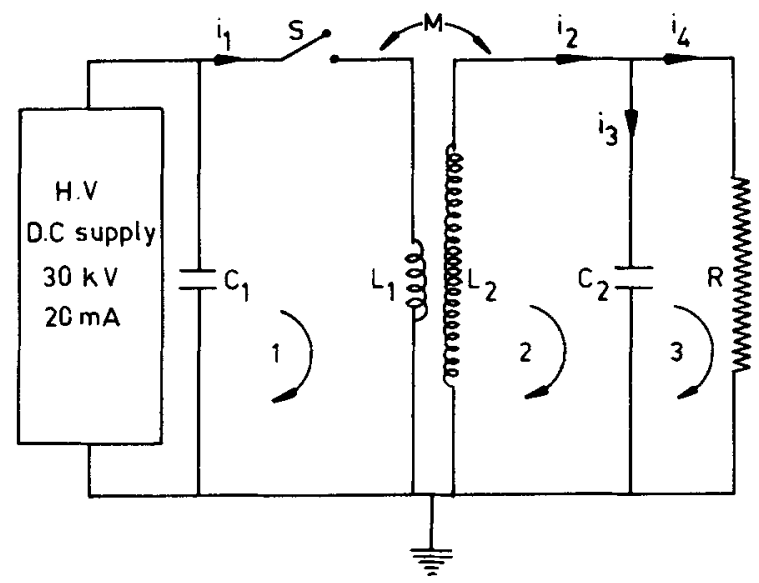

Figure 2. Equivalent circuit of a Tesla transformer.

Laplace transforming (1)-(4), solutions for primary and secondary currents and voltages are obtained (Cook \& Reginato 1979), which are:

$$
\begin{aligned}
i_{1}(t)= & {\left.\left[V_{0} / L_{1}(A)^{1 / 2}\right]\left\{\left[\omega_{2}^{2}-s_{1}^{2}\right) / s_{1}\right] \sin s_{1} t-\left[\left(\omega_{2}^{2}-s_{2}^{2}\right) / s_{2}\right] \sin s_{2} t\right\} } \\
i_{2}(t)=- & {\left[V_{0} k /\left(L_{1} L_{2}\right)^{1 / 2}(A)^{1 / 2}\right]\left[s_{2} \sin s_{2} t-s_{1} \sin s_{1} t\right] } \\
V_{1}(t)= & {\left[V_{0} \omega_{1}^{2} /(A)^{1 / 2}\right]\left(L_{2} / L_{1}\right)^{1 / 2}\left\{\left[\left(\omega_{2}^{2}-s_{1}^{2}\right) / s_{1}^{2}\right] \cos s_{1} t\right.} \\
& \left.-\left[\left(\omega_{2}^{2}-s_{2}^{2}\right) / s_{2}^{2}\right] \cos s_{2} t\right\}
\end{aligned}
$$

and

$$
V_{2}(t)=\left[V_{0} k \omega_{2}^{2} /(A)^{1 / 2}\right]\left(L_{2} / L_{1}\right)^{1 / 2}\left[\cos s_{2} t-\cos s_{1} t\right]
$$

where

and

$$
\begin{aligned}
s_{1}^{2}, s_{2}^{2} & =\left\{\left(\omega_{1}^{2}+\omega_{2}^{2}\right) \pm\left[\omega_{1}^{4}+\omega_{2}^{4}-2\left(1-2 k^{2}\right) \omega_{1}^{2} \omega_{2}^{2}\right]^{1 / 2}\right\} /\left[2\left(1-k^{2}\right)\right] \\
(\mathrm{A})^{1 / 2} & =\left[\omega_{1}^{4}+\omega_{2}^{4}-2\left(1-2 k^{2}\right) \omega_{1}^{2} \omega_{2}^{2}\right]^{1 / 2}, \\
\omega_{1} & =1 /\left(L_{1} C_{1}\right)^{1 / 2}, \\
\omega_{2} & =1 /\left(L_{2} C_{2}\right)^{1 / 2} .
\end{aligned}
$$

Theoretically, $100 \%$ transfer of energy from the primary capacitor $C_{1}$ to the secondary capacitor $C_{2}$ takes place at the instant when secondary voltage is maximum and primary voltage and current and secondary current are zero (Hoffmann 1975; Boscolo et al 1975; Cook \& Reginato 1979).

Voltage and current waveforms for different coupling co-efficients $K$ and for various tuning ratios $W_{1} / W_{2}$ can be generated from (5)-(8). It is found from these waveforms that $100 \%$ energy transfer from $C_{1}$ to $C_{2}$ will take place only if (i) the resonant frequency of the primary circuit $W_{1}$ with the secondary open is equal to the resonant frequency of the secondary circuit $W_{2}$ with the primary open and (ii) the coupling coefficient $K$ has discrete values like $0.6,0.385,0.27,0.153$ etc.

Under these two conditions, energy stored in the primary $L C$ circuit at time $t=0$ will be transfered to the secondary $L C$ circuit, at an instant later $t=t_{\mathrm{p}}$ with primary and secondary currents zero. The coupled primary and secondary circuits have frequencies in the ratio 2:1 under these conditions. Hence, it is often called the double resonance 
(Finkelstein et al 1966) principle of operation of the Tesla transformer. Primary and secondary voltages and currents for the resonant case, i.e. $W_{1}=W_{2}$ and $K=0.6$, are shown in figure 3.

Case $B$ when $R$ is finite: In this case, the Laplace transform of (1)-(4) will give us:

$$
\begin{aligned}
& V_{0} / s=I_{1}(s)\left[L_{1} s+\left(1 / c_{1} s\right)\right]-I_{2} M s \\
& O=I_{2} L_{2} s+\left(I_{3} / c_{2} s\right)-I_{1} M s \\
& I_{3} / C_{2} s=R I_{4}(s)
\end{aligned}
$$

and $\quad I_{2}(s)=I_{3}(s)+I_{4}(s)$.

Assuming $W_{1}=1 /\left(L_{1} C_{1}\right)^{1 / 2}=1 /\left(L_{2} C_{2}\right)^{1 / 2}=W_{2}=W$ (say) and solving (9)-(12) for $I_{2}$ and $V_{2}$ we have:

$$
\begin{aligned}
I_{2}(s)= & {\left[V_{0} K /\left(1-k^{2}\right)\left(L_{1} L_{2}\right)^{1 / 2}\right]\left[s^{2}+L_{2 / R} \omega_{s}^{2}\right] /\left\{s^{4}+\left(L_{2} / R\right) \omega^{2} s^{3}\right.} \\
& \left.+\left[2 \omega^{2} /\left(1-k^{2}\right)\right] s^{2}+\left[2 \omega^{4} / R\left(1-k^{2}\right)\right] s+\omega^{4} /\left(1-k^{2}\right)\right\},
\end{aligned}
$$

and

$$
\begin{aligned}
V_{2}(s)= & {\left[V_{0} k / c_{2}\left(1-k^{2}\right)\left(L_{1} L_{2}\right)^{1 / 2}\right] s /\left\{s^{4}+\left(L_{2} / R\right) \omega^{2} s^{3}\right.} \\
& \left.+\left[2 \omega^{2} /\left(1-k^{2}\right)\right] s^{2}+\left[2 \omega^{4} / R\left(1-k^{2}\right)\right] s+\omega^{4} /\left(1-k^{2}\right)\right\} .
\end{aligned}
$$

The denominator of the above equations contains a fourth order algebraic equation. Its roots were obtained numerically using Bairstow's method and then inverse Laplace transformed with the help of a standard table (Bateman 1954, p. 232). Time variation of secondary output voltage across capacitor $C_{2}$ for different values of secondary loss resistance $R$ is shown in figure 4. It is noteworthy from the figure that a finite value of $R$ reduces amplitude of secondary voltage, however, the time variation is not affected (compare with figure 3). When $R C_{2}$ is comparable to natural frequency secondary or primary circuit (because $W_{1}=W_{2}$ ), $V_{2}$ is decreased by $55 \%$ and thus energy transfer
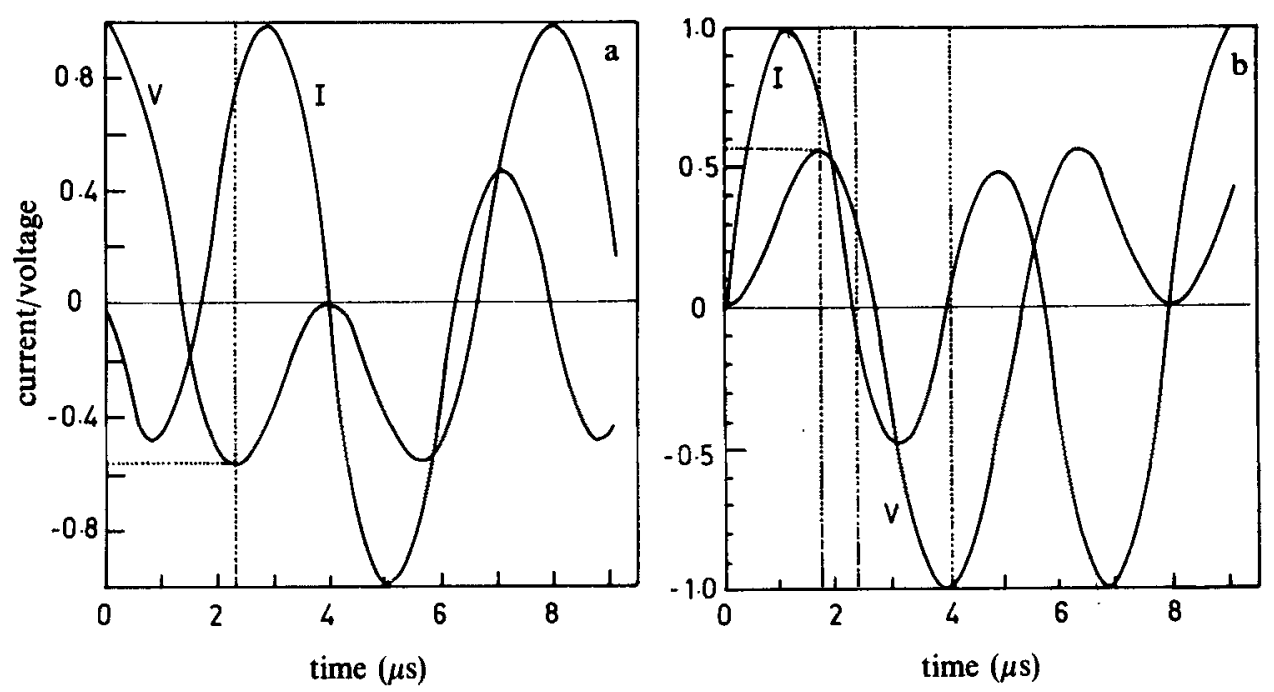

Figure 3a. Primary voltage and secondary current vs. time. b. Secondary voltage and primary current vs. time. $\left(K=0.6000, W_{1} / W_{2}=1 \cdot 0\right.$. $)$ 

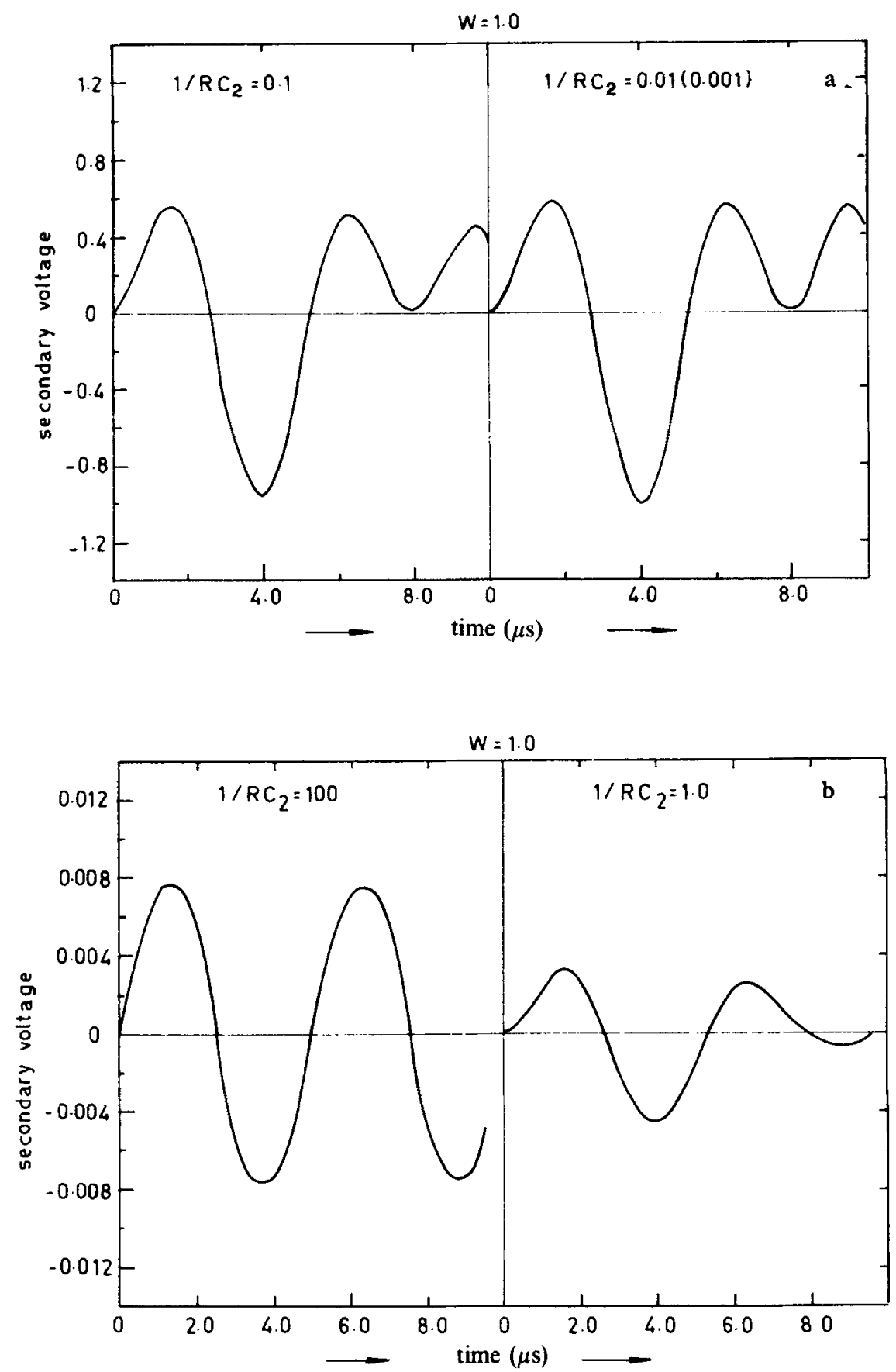

Figure 4a. Secondary voltage waveform for $R C_{2}>W$. b. Secondary voltage vs. time for $R C_{2}<W$.

efficiency by $30 \%$. For the case of $R C_{2} \ll W$, secondary capacitor $C_{2}$ charges to very low voltage. Therefore, for getting high energy transfer efficiency, a large value of secondary loss resistance is desired. 


\subsection{Design and construction}

The Tesla transformer was designed for peak secondary output voltages of $900 \mathrm{kV}$ with a voltage step-up ratio of 30 . Further, to obtain maximum energy transfer efficiency from the primary capacitor to the secondary capacitor, the double resonance principle of operation was chosen. The choice of a double resonance mode of operation implies that the resonant frequency of the primary circuit for open (uncoupled) secondary circuit must equal the resonant frequency of the secondary for an open primary, and the coupling co-efficient $K$ must have one of these values $(0 \cdot 6,0 \cdot 385,0 \cdot 27,0 \cdot 15$ etc.). It is also desirable to have short charging time of the pulse forming line since the electric breakdown of the dielectric of the pulse forming line is inversely proportional to the third root of the charging time (Nation 1979). Because secondary output voltage takes the minimum time to reach its peak value for $K=0 \cdot 6$, this value of the coupling co-efficient was chosen.

Two types of windings are generally used (Nagesh 1982, p. 237) for construction of a Tesla transformer, viz, (i) helical winding, and (ii) spiral winding. In case of helical winding, the secondary winding is on an insulated former in the axial direction. The primary winding covers the secondary winding completely. In case of spiral winding, the primary and secondary windings are on an insulated former in the radial direction. The spiral type of winding was chosen for the present case as it possesses the following advantages over the helical one:

(i) high co-efficient of coupling can be obtained and hence high efficiency;

(ii) the secondary windings are capacitively graded and so there is no necessity of guard rings to protect the secondary;

(iii) the system is compact.

The design of the Tesla transformer is dependent on the co-efficient of coupling, voltage step-up ratio, electrical strength requirement and availability of capacitors. The design for a given secondary output voltage and coupling co-efficient is an iterative process and was started by assuming values for the step-up ratio and the primary input voltage which in turn fixed the ratio $L_{1} / L_{2}$. The insulation thickness between the turns was chosen to satisfy electrical breakdown strength requirements. The radii of windings and the number of primary and secondary turns were systematically adjusted until the calculated values of inductance and coupling co-efficient were acceptable. The choice of charging time for the pulse forming line fixed the primary and secondary capacitors.

The primary of the Tesla transformer is a two-turn coil made of $0.3 \mathrm{~mm}$ thick and $50.0 \mathrm{~cm}$ wide copper sheet and is wound on a polythene cylinder of $400 \mathrm{~mm}$ diameter; and has a design inductance of $1.0 \mu \mathrm{H}$. The insulating dielectric betwoen the windings consists of nine layers of polythene with a total thickness of $2.7 \mathrm{~mm}$. The secondary is a 57-turn coil surrounding the primary. The secondary coil has the inner diameter $\phi$ $=406 \mathrm{~mm}$ and is made of $0.1 \mathrm{~mm}$ thick and $500 \mathrm{~mm}$ wide aluminium foil. The secondary has a $0.85 \mathrm{mH}$ inductance. The step-up transformer is placed in a mild steel tank which is then filled with insulating transformer oil. An arrangement for primary and secondary winding is shown in figure 5 , while the cross-sectional view is depicted in figure 6.

The primary energy is stored in three Maxwell capacitors connected in parallel, having a total capacitance of $7 \cdot 2 \mu \mathrm{F}$. Maximum energy storage capability of the capacitor bank is $3.2 \mathrm{~kJ}$ at $30 \mathrm{kV}$. The primary spark gap is made of brass electrodes with trigger facility (Trigatron type). The trigger electrode is coupled to the high voltage 


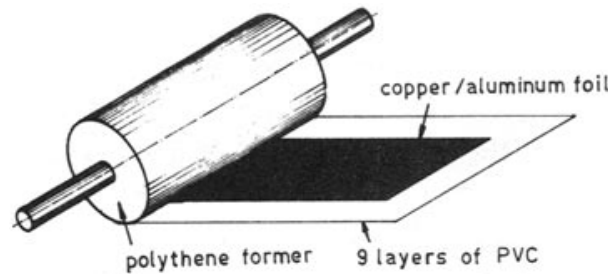

Figure 5. An arrangement for primary and secondary winding of the transformer.

trigger pulse by a helical wire line having $10 \Omega$ characteristic impedance. When the spark gap fires, the inductance of the helix decouples the spark gap electrode from the ground. The spark gap can be operated in a 8 to $30 \mathrm{kV}$ voltage range by adjusting the gas pressure in the gap.

\section{Pulse forming line}

A coaxial line which acts as the secondary capacitor during resonance charging with water dielectric is used as the pulse forming line (PFL) to get short pulse lengths. The choice of water as the dielectric $(\varepsilon=80)$ permits compact construction with high energy density. Water has the advantage of being able to recover from a breakdown without having formed conducting byproducts.

The coaxial water line consists of an inner conductor of $150 \mathrm{~mm}$ diameter and an outer one of $300 \mathrm{~mm}$. The length of the coaxial line is $800 \mathrm{~mm}$. The inner and outer conductor diameters of the pulse forming line have been determined from the capacitance requirement and the allowable electrical stress at which the dielectric can

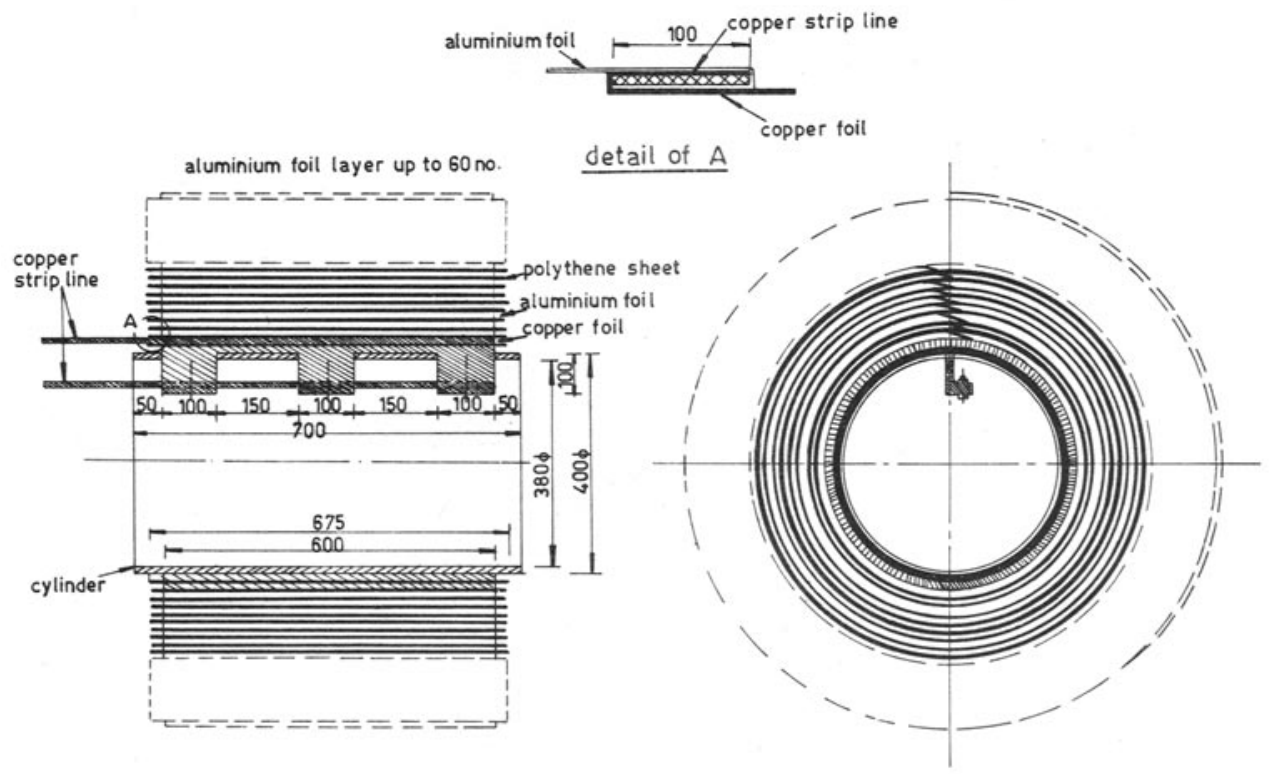

Figure 6. Cross-sectional view of the Tesla transformer. 
operate. The choice of output pulse duration fixes the line length. Both the conductors of the line are made of stainless steel to maintain the high resistivity required for water.

Perspex stand-offs are used to support the inner conductor. Because of the longer charge time characteristics of the Tesla transformer, the problems of losses due to finite resistivity (see \$2.1) and water breakdown are of greater concern than with the Marxcharged system. Water resistivity of $1.0 \mathrm{M} \Omega \mathrm{cm}$ is required for satisfactory operation, up to $800 \mathrm{kV}$. High purity bubble free water is also required to prevent premature breakdown in the coaxial line. The water resistivity is maintained at around $1 \mathrm{M} \Omega \mathrm{cm}$ with the help of commercially available deioniser (Indian Ion Exchange Ltd., Model cv 20 and M6) which corresponds to a total resistance of $2.0 \mathrm{k} \Omega$. The pulse forming line has a capacitance of $5 \cdot 1 \mathrm{nF}$, a pulse length of $50 \mathrm{~ns}$ and a characteristic impedance of $4.8 \Omega$.

\section{High voltage switch and field emission diode}

The high voltage switch has been designed for discharging the pulse forming line, at peak charging voltage, into the field emission diode. The spark gap is made of brass electrodes housed in a sealed perspex tube having circular grooves on the inner surface to avoid surface breakdown. The clearance between the facing tips of the electrodes is chosen as $5 \mathrm{~cm}$. The breakdown voltage is adjusted by charging the pressure of the $\mathrm{N}_{2} / \mathrm{SF}_{6}$ gas. The spark gap is designed for the overvoltage self trigger mode of operation and can be operated in a $150-800 \mathrm{kV}$ voltage range.

The design of the field emission diode (which converts the high voltage pulse into an electron beam) is dictated by the choice of the injection technique to be used for launching the electron beam into the toroidal device. At present, a drift technique (Benford et al 1973; Mohri et al 1979, p. 311), has been chosen for injecting the intense beam into the BETA device. The idea is to launch the beam parallel to the toroidal field $B_{T}$ from one of the radial ports. Curvature and gradient drift move the beam along the direction of major axis. Beam drift continues until it reaches the chamber surface unless additional forces are applied to stabilise the motion at a suitable distance from the injector. Since the curvature drift is actually an $\mathbf{F}_{c} \times \mathbf{B}_{T}$ drift, $F_{c}$ being the centrifugal force, we need only apply a counteracting radial force to stop the drift. By applying an external magnetic field with the predominant component $B_{2}$ parallel to the major axis, beam drift will be stabilised. In the BETA device the field emission diode is located $5 \mathrm{~cm}$ inside the outer vacuum wall on the equitorial plane of the torus, as shown in figure 1. It consists of a brass cathode, having a $4 \mathrm{~cm}$ dia emitting surface, and a grid anode. The diode is positioned in such a way that it ejects electrons parallel to the toroidal magnetic field. A Rogowskii coil is placed over the cathode shank to monitor the diode current.

\section{Characteristics of operation}

A number of experiments were carried out to establish the characteristics of the electron beam generator. First of all we measured the charging voltage and its wave form for the pulse forming line by a resistive divider installed at the secondary terminal of the Tesla transformer. Figure 7 shows a typical voltage waveform when the secondary spark gap did not break down. The maximum voltage appears at the second peak as designed. The voltage step-up ratio of the Tesla transformer is observed to be strongly dependent on 


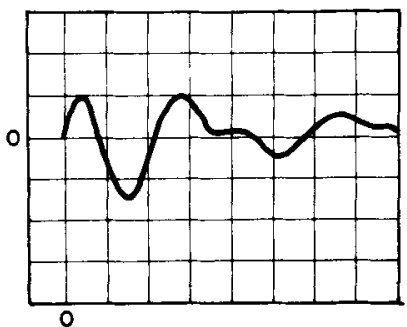

Figure 7. Waveform of the secondary voltage when the high voltage holding switch does not fire (horizontal scale: $5 \mu \mathrm{s} /$ div; vertical scale: $100 \mathrm{kV} / \mathrm{div}$ ).

the quality of water in the PFL. In the best condition when the specific resistance of the water is greater than $1.0 \mathrm{M} \Omega \mathrm{cm}$, the step-up ratio is 25 . The efficiency of the energy transfer from the primary capacitor bank to the pulse forming line is $44 \%$. A comparison of the designed and measured characteristic parameters of the Tesla transformer is made in table 1 . The discrepancy between the designed and the observed efficiency of the Tesla transformer is due perhaps to three factors. First, the series resistance in the primary and secondary circuits are larger than the designed value which results in low $Q$ values of these circuits and hence, low efficiency. Second, the Tesla transformer is housed in a mild steel tank in which induced current flows. The induced wall current causes a reduction in the magnetic flux coupling the secondary winding, this lowers step-up voltage ratio. Lastly, the resistance of the water in the coaxial line is small $(2.0 \mathrm{k} \Omega)$ as compared to designed value $(5.0 \mathrm{k} \Omega)$ resulting in low energy transfer efficiency from primary capacitor bank to secondary coaxial water line capacitance.

The gas pressure of the high voltage switch is adjusted such that the gas breaks down at the second peak of the charged voltage of the pulse forming line. Figure 8 shows the measured Tesla transformer output voltage waveform, when the high voltage switch breaks down at the second peak.

As mentioned in the last section, the diode current was monitored with a Rogowskii coil placed over the cathode shank. Figure 9 represents a typical current waveform of the diode which is placed inside the BETA device for beam injection. The diode current rises to $14 \mathrm{kA}$ in $80 \mathrm{~ns}$ time and its pulse width is about $150 \mathrm{~ns}$.

\section{Conclusion}

A Tesla transformer type relativistic electron beam generator has been fabricated and operated successfully. It is found that the Tesla transformer can efficiently transfer the energy stored in the primary capacitor bank to the pulse forming line. An electron beam of $100-200 \mathrm{keV}$ energy with $20 \mathrm{kA}$ current lasting for $150 \mathrm{~ns}$ has been obtained.

Table 1. A comparison of designed and measured parameters of the Tesla transformer

\begin{tabular}{lcc}
\hline Parameter & Designed value & Measured value \\
\hline Coupling co-efficient & 0.6 & 0.56 \\
Time for peak voltage & $8.22 \mu \mathrm{s}$ & $7.5 \mu \mathrm{s}$ \\
Voltage step-up ratio & $30 \cdot 00$ & 25.00 \\
Energy transfer efficiency & $64 \%$ & $44 \%$ \\
\hline
\end{tabular}




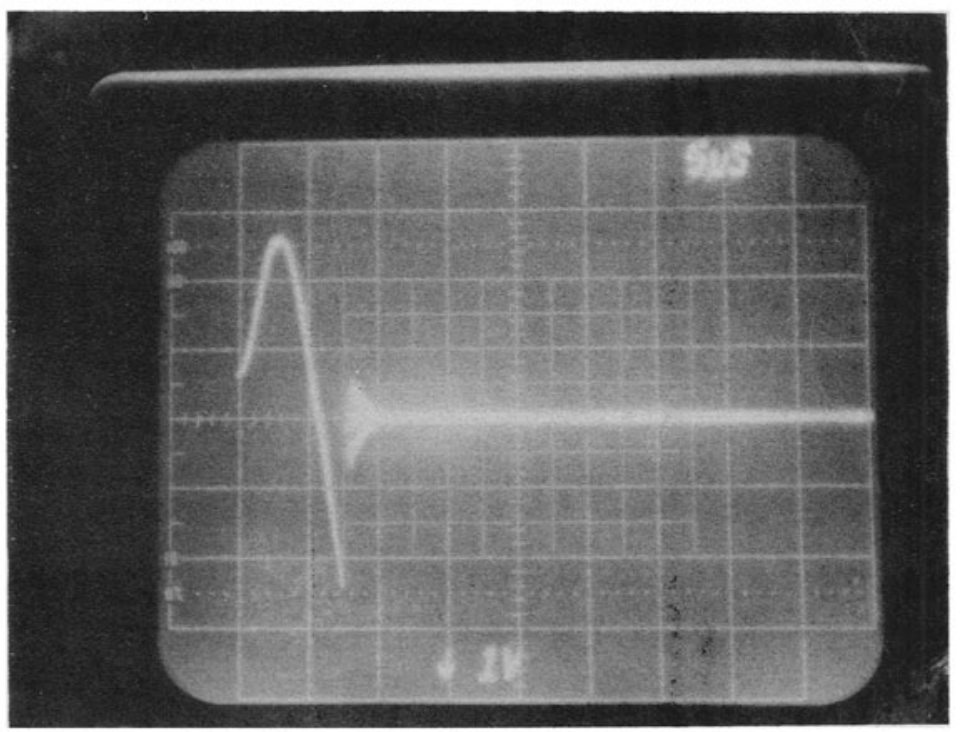

Figure 8. Oscillogramme of the secondary voltage waveform when high voltage holding switch fires (horizontal scale: $5 \mu \mathrm{s} / \mathrm{div}$; vertical scale: $50 \mathrm{kV} / \mathrm{div}$ ).

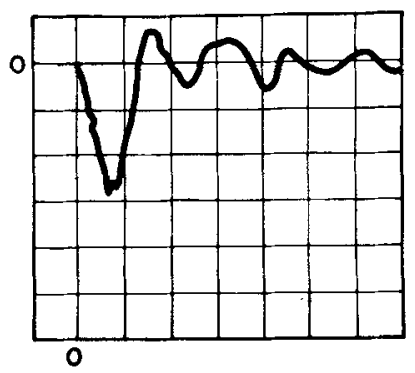

Figure 9. A typical diode current waveform (horizontal scale: $100 \mathrm{~ns} / \mathrm{div}$; vertical scale: $5 \mathrm{kA} / \mathrm{div}$ ).

The authors would like to express sincere thanks to the members of the plasma physics programme for their help during the fabrication stage.

\section{References}

Bateman H 1954 Table of integral transforms (New York: McGraw Hill) vol. 1

Benford J, Putnam S, Stallings C, Guillory J, Shope S 1973 Phys. Rev. Lett. 31: 346-349

Boscolo I, Brautti G, Coisson R, Leo M, Luches A 1975 Rev. Sci. Instrum. 46: 1535-1538

Cook E G, Reginato L L 1979 IEEE Trans. Electron Devices ED-26: 1512-1517

Finkelstein D, Goldberg P, Schuchatowitz J 1966 Rev. Sci. Instrum. 37: 159-162

Hoffmann C R J 1975 Rev. Sci. Instrum. 46: 1-4

Jain K K, John P I 1984a Plasma Phy. 26: 891-905

Jain K K, John P I 1984b Pramana (J. Phys.) 23: 1-16

Kapetanakos C A, Black W M, Striffler C D 1975 Appl. Phys. Lett. 26: 368-370

Korn P, Sandel F, Wharton C B 1973 Phys. Rev. Lett. 31: 579-583 
Mohri A, Narihara K, Tsuzuki T, Kubota Y, Tomita Y, Ikuta K, Masuzaki M 1979 Plasma physics and controlled nuclear fusion research 1978 (Vienna: IAEA) vol. 3

Nation J A 1979 Part. Accl. 10: 1-30

Nagesh K V 1982 Proc. technology of electric insulation and high voltage pulse techniques (Bombay: Bhabha Atomic Research Centre)

Olsen C 1975 Phys. Fluids 18: 585-597, 598-606

Roberson C W, Eckhouse S, Fisher A, Robertson S, Rostoker N 1976 Phys. Rev. Lett. 36: 1457-1460

Roberson C W 1978 Nucl. Fusion 18: 1693-1704

Sethian J D, Gerber K A, Spector D N, Robson A E 1978 Phys. Rev. Lett. 41: 798-801

Sprangle P, Drobot A, Manheimer W 1976 Phys. Lett. 36: 1180-1183

Yonas G 1983 Plasma physics and controlled nuclear fusion 1982 (Vienna: uEA) vol. 2

Yonas G, Poukey J W, Prestwich K R, Freeman J R, Toepfer A J, Clauser M J 1974 Nucl. Fusion 14: 731-740 